Article

\title{
Ag@Au Core-Shell Porous Nanocages with Outstanding SERS Activity for Highly Sensitive SERS Immunoassay
}

\author{
Yaqi Huang ${ }^{1}$, Dajie Lin ${ }^{1,2, *}$, Mengting Li ${ }^{1}$, Dewu Yin ${ }^{1}$, Shun Wang ${ }^{1, *}$ and Jichang Wang ${ }^{1,3, *}$ \\ 1 College of Chemistry and Materials Engineering, Wenzhou University, Wenzhou 325035, China; \\ 16451282265@stu.wzu.edu.cn (Y.H.); 17451282201@stu.wzu.edu.cn (M.L.); dewuyin@wzu.edu.cn (D.Y.) \\ 2 Key Laboratory of Laboratory Medicine (Ministry of Education of China), Wenzhou Medical University, \\ Wenzhou 325037, China \\ 3 Department of Chemistry and Biochemistry, University of Windsor, Windsor, ON N9B 3P4, Canada \\ * Correspondence: lindj@wzu.edu.cn (D.L.); shunwang@wzu.edu.cn (S.W.); jwang@uwindsor.ca (J.W.); \\ Tel.: +86-577-8668-9357 (S.W.)
}

Received: 26 February 2019; Accepted: 27 March 2019; Published: 31 March 2019

\begin{abstract}
A highly sensitive immunoassay of biomarkers has been achieved using 4-mercaptobenzoic acid-labeled Ag@Au core-shell porous nanocage tags and $\alpha$-fetoprotein immuno-sensing chips. The Ag@Au porous nanocages were uniquely synthesized by using an Ag core as a self-sacrificial template and reducing agent, where the slow reaction process led to the formation of a porous $\mathrm{Au}$ layer. The size of the remaining $\mathrm{Ag}$ core and surface roughness of the $\mathrm{Au}$ shell were controlled by adjusting the chloroauric acid concentration. The porous cage exhibited excellent surface-enhanced Raman spectroscopy (SERS) activity, presumably due to a synergetic interaction between newly generated hot spots in the rough Au shell and the retained SERS activity of the Ag core. Using $\alpha$-fetoprotein as a model analyte for immunoassay, the SERS signal had a wide linear range of $0.20 \mathrm{ng} \mathrm{mL}^{-1}$ to $500.0 \mathrm{ng} \mathrm{mL}^{-1}$ with a detection limit of $0.12 \mathrm{ng} \mathrm{mL}^{-1}$. Without the need of further signal amplification, the as-prepared Ag@Au bimetallic nanocages can be directly used for highly sensitive SERS assays of other biomarkers in biomedical research, diagnostics, etc.
\end{abstract}

Keywords: surface-enhanced Raman spectroscopy; immunoassay; biomarkers; Ag core-Au shell porous nanocages; galvanic replacement reaction

\section{Introduction}

Clinical measurements of biomarkers can greatly help the early diagnosis, prediction, treatment monitoring and guidance of various diseases such as cancer [1]. Being able to achieve very specific and accurate detection of biomarkers is no doubt crucial in many areas of biomedical research and medical diagnostics [2]. For example, $\alpha$-fetoprotein (AFP) has been developed as a biomarker for the early diagnosis of liver cancer. Generally, the AFP level exceeding the threshold of $25 \mathrm{ng} \mathrm{mL}^{-1}$ in serum is suspected to be associated with the occurrence of liver cancer or other related diseases [3]. For the above reason, in the past decade a number of novel immunosensors have been developed for cancer biomarker detection, such as enzyme-linked immunoassay [3,4], electrochemistry immunoassay [5-7], fluorescence immunoassay [8-10] and chemiluminescence immunoassay [11,12]. Although the abovementioned immunosensors have been well elaborated and some of them have even been applied in clinical trials, the development of immunoassays with better sensitivity, higher selectivity and more simplicity for rapid cancer biomarker detection in cancer patient serum is still both highly challenging and greatly needed [13]. 
Surface-enhanced Raman spectroscopy (SERS) is one of the modern techniques that are capable of the ultrasensitive detection and quantification of molecules on or near the surface of plasmonic nanostructures [14,15], and has wide applications in immunoassays (SERS-based immunoassays). In the process of SERS spectroscopy, the high selectivity and resolution of SERS spectra are key factors that are superior to traditional infrared and fluorescence spectroscopy. However, whether SERS-based immunoassays can be effectively employed in clinics depends on their detection sensitivity [15]. In order to achieve high sensitivity, new technical methods of fabricating the platform have been used. For example, compared with naked Au nanoparticles, enzyme-induced silver deposition could enhance the SERS signal four times [16]. Multiple SERS tags, attaching intrinsically strong Raman scattering molecules (called Raman reporters) to the surface of plasmon-resonant nanoparticles, binding to different epitopes on a protein biomarker was also reported to improve the detection sensitivity [17]. It is clear that the efficiency of detection was affected by the preparation steps. As the SERS effect is related to the surface roughness of the substrate [18], a new class of multi-branched nanostructures such as nanostars $[19,20]$ and nanoflowers [21], which have a higher surface roughness than spherical particles of similar size, has been synthesized. In addition to these monometallic nanostructures mentioned above, multi-component nanostructures such as bimetallic core-shell nanoparticles [22], nanourchins [14] and satellite nanostructures [23] have also attracted increasing attention [24].

In this work, Ag@Au core-shell porous nanocages were synthesized through galvanic replacement reactions using an Ag nanoparticle as a self-sacrificial template. The as-prepared nanostructures exhibit excellent SERS activity, a desired property of SERS tags to develop highly sensitive SERS immunosensors (Scheme 1). In order to maximize the sensitivity of the SERS signal, the size of the Ag core and surface roughness of the porous Au shell were optimized by adjusting the amount of chloroauric acid added to the solution that contained Ag nanoparticles with diameters of $50 \pm 5 \mathrm{~nm}$. Using numerical differentiation (DDA), it was calculated that the enhancement of the electromagnetic field generated by the active sites around the nanopores can be more than 10 times higher than that at other sites [25], implying that the Ag@Au porous nanocage prepared in this study can provide a plentiful number of hot spots with enormously enhanced SERS activity. The excellent SERS activity of the Ag@Au core-shell porous nanocage SERS tags led to ultrahigh sensitivity, in which $\alpha$-fetoprotein (AFP) could be detected down to $0.12 \mathrm{ng} \mathrm{mL}^{-1}$ without the need of further signal amplification.

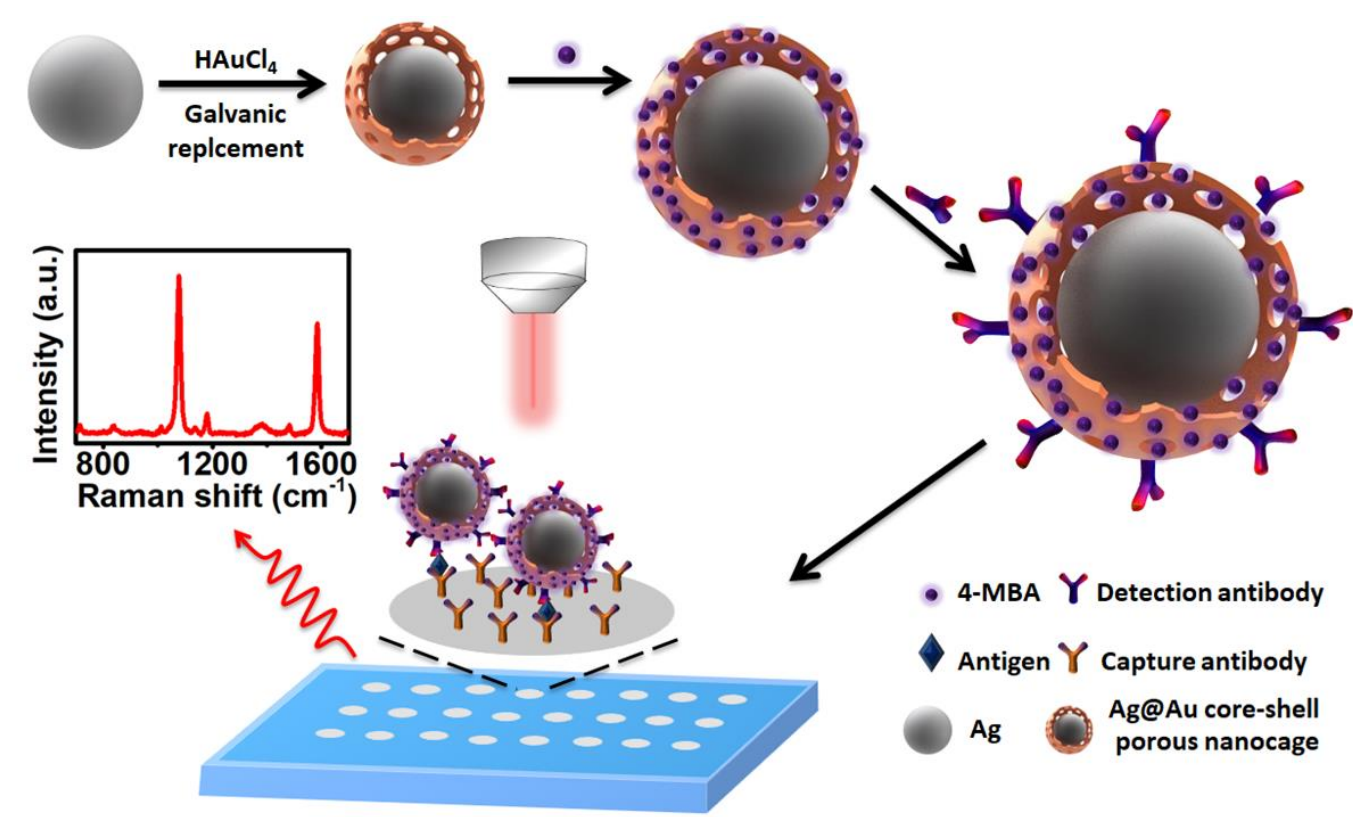

Scheme 1. Scheme of sandwich assays using 4-mercaptobenzoic acid (4-MBA) labeled Ag core-Au shell nanocage surface-enhanced Raman spectroscopy (SERS) tags modified with $\alpha$-fetoprotein (AFP) detection antibodies and AFP immuno-sensing chips. 


\section{Materials and Methods}

\subsection{Chemical Reagents}

Silver nitrate $\left(\mathrm{AgNO}_{3}, 99.8 \%\right)$ was purchased from Shanghai Chemical Reagent Co., Ltd. (China). Sodium citrate dihydrate (99.0\%), ascorbic acid $(99.99 \%)$, chloroauric acid tetrahydrate (99.9\%), 4-mercaptobenzoic acid (4-MBA), bovine serum albumin (BSA), (3-aminopropyl) triethoxysilane (APTES, 96\%) and glutaraldehyde (50\% wt in $\mathrm{H}_{2} \mathrm{O}$ ) were purchased from Aladin. Capture antibody $\left(\mathrm{Ab}_{1}\right)$ and detection antibody $\left(\mathrm{Ab}_{2}\right)$ of AFP (clone no. A2 and $\mathrm{A} 4$ ) were purchased from Beijing Bioss

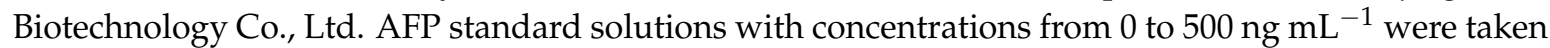
from ELISA kits of AFP, which were obtained from Fujirebio Diagnostics AB (Göteborg, Sweden). Ultrapure water prepared with a Millipore water purification system (18 M $\Omega$, Milli-Q, Millipore) was used in all assays. Phosphate buffer (PBS, $0.01 \mathrm{M})$ of various pHs was prepared by mixing the stock solutions of $\mathrm{NaH}_{2} \mathrm{PO}_{4}$ and $\mathrm{Na}_{2} \mathrm{HPO}_{4}$. The washing buffer was PBS (0.01 M, pH 7.4) containing $0.02 \%$ (w/v) Tween-20 (PBST). All other reagents were of analytical grade and used as received.

\subsection{Synthesis of Ag Nanoparticles}

Ag nanoparticles (Ag NPs) were prepared through the reduction of $\mathrm{AgNO}_{3}$ by ascorbic acid and sodium citrate under chloride ion induction [26]. Ag NPs of $50 \pm 5 \mathrm{~nm}$ in diameter were obtained by using the above method. First, $1.0 \mathrm{~mL}$ of $34.0 \mathrm{mM}$ sodium citrate and $30.0 \mu \mathrm{L}$ of $0.1 \mathrm{M} \mathrm{NaCl}$ solution were successively added to $2.4 \mathrm{~mL}$ of $0.01 \mathrm{M} \mathrm{AgNO}_{3}$ solution, then mixed. After 5 minutes, the mixture was rapidly added to $46.6 \mathrm{~mL}$ of boiling water that contained $100.0 \mu \mathrm{L}$ of $58.3 \mathrm{mM}$ ascorbic acid, with the ascorbic acid being added 1 minute prior to the addition of the mixture. Finally, the solution was maintained at the boiling point for $1 \mathrm{~h}$, then cooled to room temperature while being stirred to obtain the hydrosol of Ag nanoparticles. The obtained hydrosol was centrifuged at 10,000 rpm and the precipitates were re-dispersed in water. The Ag NPs hydrosol was filtered through a $0.22 \mu \mathrm{m}$ filtration membrane.

\subsection{Synthesis of Ag@Au Core-Shell Porous Nanocages}

At room temperature, $6.0 \mathrm{~mL}$ of the hydrosol of Ag nanoparticles was added to a glass bottle and ultrasonicated for $30 \mathrm{~min}$. After that, $0.253 \mathrm{mM}$ chloroauric acid solution was slowly added via a peristaltic pump while the mixture was continuously stirred for $30 \mathrm{~min}$. Different volumes of chloroauric acid solution $(0.5,1.0,1.5$ and $2.0 \mathrm{~mL})$ were studied. The Ag@Au core-shell porous nanocages hydrosol was filtered through a $0.22 \mu \mathrm{m}$ filtration membrane.

\subsection{Preparation of SERS Tags}

First, $30.0 \mu \mathrm{L}$ of $1.0 \mathrm{mM}$ 4-MBA in ethanol was added to the hydrosol of the Ag@Au core-shell porous nanocages using $1.0 \mathrm{~mL}$ of $0.253 \mathrm{mM}$ chloroauric acid aqueous solution prepared by stirring for $1 \mathrm{~h}$. Then, $200.0 \mu \mathrm{L} 20.0 \mathrm{ug} \mathrm{mL}{ }^{-1} \mathrm{Ab}_{2}$ was added to the mixture while stirring for $6 \mathrm{~h}$ at $4{ }^{\circ} \mathrm{C}$. Afterward, $100.0 \mu \mathrm{L}$ of blocking buffer containing $5 \%$ BSA and $0.02 \%$ Tween-20 was added to the mixture while stirring for $2 \mathrm{~h}$, followed by aging without stirring for $12 \mathrm{~h}$ at $4{ }^{\circ} \mathrm{C}$. The obtained Ag@Au core-shell porous nanocage SERS tags were centrifuged at $9000 \mathrm{rpm}$ and the precipitates were dispersed in $0.01 \mathrm{M}$ PBS solution. Similarly, the same procedures were used for the synthesis of Au nanoparticle SERS tags for comparison.

\subsection{Preparation of Immuno-Sensing Chips}

Quartz chips were placed into piranha solution $\left(\mathrm{H}_{2} \mathrm{O}_{2} / \mathrm{H}_{2} \mathrm{SO}_{4}, 1: 3, \mathrm{v} / \mathrm{v}\right)$ for $1 \mathrm{~h}$, then rinsed with water and dried with nitrogen gas. The cleaned quartz chips were then placed into anhydrous ethanol containing $5.0 \mathrm{mM}$ (3-aminopropyl) triethoxysilane APTES for $12 \mathrm{~h}$. It was combined with APTES via $\mathrm{O}-\mathrm{Si}-\mathrm{O}$ covalent bonds to form an aminated self-assembled monolayers. The modified chips were 
washed thoroughly with anhydrous ethanol and dried with nitrogen gas afterwards. Polyethylene terephthalate film with an array of $3 \mathrm{~mm}$ diameter holes was carefully applied to the above modified quartz chip. Next, $5.0 \mu \mathrm{L}$ of $2.5 \%$ glutaraldehyde solution was pipetted into each well and kept there for $2 \mathrm{~h}$ at room temperature before being rinsed with washing buffer. Afterward, $10.0 \mu \mathrm{L}$ of $\mathrm{Ab}_{1}$ was pipetted into each well and incubated for $4 \mathrm{~h}$ at room temperature before being rinsed with water. Subsequently, the blocking buffer that was diluted 10 times was pipetted onto the chips and incubated for $1 \mathrm{~h}$. The AFP immuno-sensing chips were then rinsed with washing buffer and stored in a plastic centrifuge tube in a nitrogen atmosphere.

\subsection{Sandwich Immunoassay}

The AFP solution of different concentrations was pipetted onto the immuno-sensing chips and incubated for $30 \mathrm{~min}$ at $37{ }^{\circ} \mathrm{C}$. The modified chips were then rinsed with washing buffer. The Ag@Au core-shell porous nanocage SERS tags were subsequently pipetted onto the AFP-captured immuno-sensing chips and incubated for $30 \mathrm{~min}$ at $37^{\circ} \mathrm{C}$. Finally, the un-captured Ag@Au core-shell porous nanocages were washed away with washing buffer. The chips were then dried under nitrogen protection prior to SERS measurement.

\subsection{Instruments and Measurements}

Transmission electron microscopy (TEM) images were obtained with a JEOL JEM-2100 high-resolution transmission electron microscope, using an accelerating voltage of $200 \mathrm{kV}$. Ultraviolet-visible (UV-vis) spectra of the samples was obtained with a Shimadzu ultraviolet spectrophotometer (UV-2450). Raman spectra were recorded on a Renishaw inVia Raman spectrometer equipped with a charge coupled device (CCD) detector. The excitation lasers with a wavelength of $785 \mathrm{~nm}$ were used for measurements. SERS spectra were acquired using two platforms: (1) SERS spectra of 4-MBA-labeled Ag@Au core-shell nanocages, where the typical exposure time for each measurement was $10 \mathrm{~s}$ with one-time accumulation and the laser power was set to $10 \mathrm{~mW}$; and (2) sandwich assays between the SERS tags and the immuno-sensing chips, where the typical exposure time for each measurement was $20 \mathrm{~s}$ with one-time accumulation and the laser power was set to $10 \mathrm{~mW}$. All of the SERS spectra shown in this report were baseline-corrected, but not normalized.

\section{Results and Discussion}

\subsection{Characterization of Ag Core-Au Shell Nanocages}

$\mathrm{Ag}$ NPs with a diameter of $50 \pm 5 \mathrm{~nm}$ were first synthesized with $\mathrm{AgNO}_{3}$, ascorbic acid and sodium citrate under chloride ion induction. The localized surface plasmon resonance (LSPR) band of the as-synthesized Ag NPs appeared at $415 \mathrm{~nm}$, and the maximum absorption gradually shifted to $440 \mathrm{~nm}$, accompanied by a decrease in intensity. Ag@Au core-shell porous nanocages were readily synthesized through galvanic replacement reactions using Ag NPs as the sacrificial templates. The Ag absorption band was broadened upon the increase of the amount of chloroauric acid added, owing to the formation of the Au shell on the surface of the Ag core and the superposition of their LSPR band (Figure 1). Upon continuous increase of chloroauric acid, a new band appeared in the range of 500-700 nm, presumably due to the LSPR absorption of the Au shell. The LSPR band of Ag NPs was progressively weakened, indicating that the Ag core had been gradually replaced and oxidized by $\mathrm{HAuCl}_{4}$. Phenomenologically, the corresponding hydrosols underwent a series of distinct color changes from yellowish green to reddish brown, purple and blue (Figure 1a-e). 


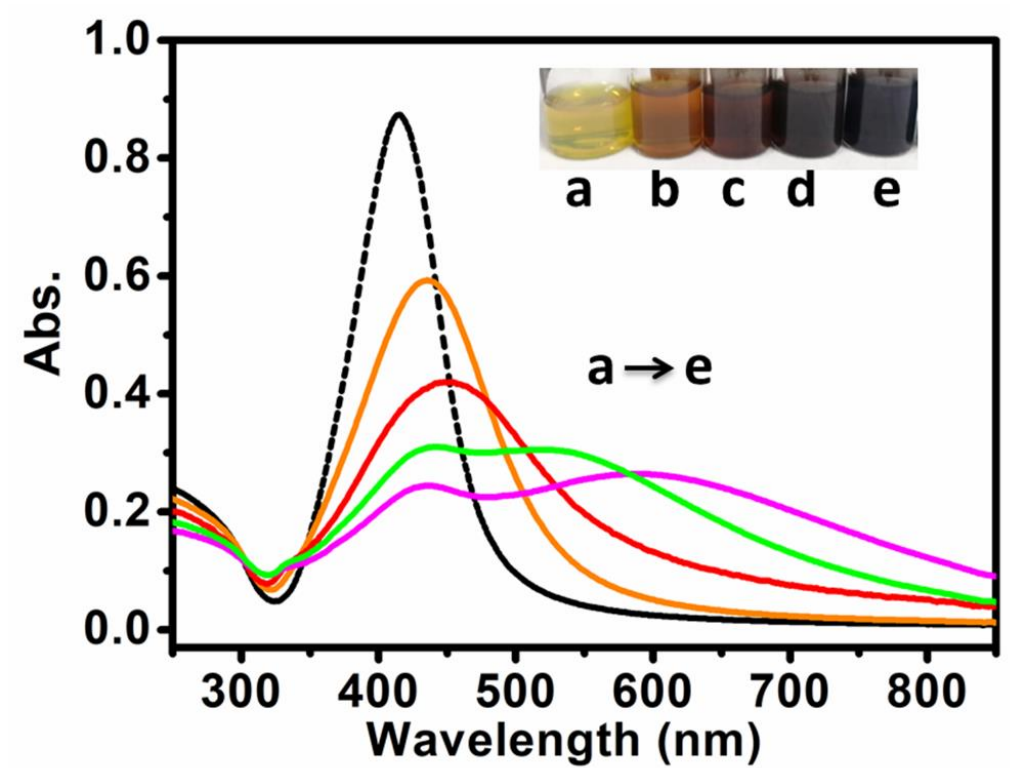

Figure 1. UV-vis spectra of the hydrosols of Ag nanoparticles and Ag core-Au shell nanocages, corresponding to different amounts of chloroauric acid aqueous solution $(0.253 \mathrm{mM})$ added $(\mathrm{mL})$ : (a) 0.0 ; (b) 0.5 ; (c) 1.0 ; (d) 1.5 ; (e) 2.0. Inset shows the corresponding photographic images.

As shown in Figure 2A, the nearly uniform Ag@Au core-shell porous nanocages have diameters of $50 \pm 5 \mathrm{~nm}$. The highly matched lattice constant of $\mathrm{Ag}(0.409 \mathrm{~nm})$ and $\mathrm{Au}(0.408 \mathrm{~nm})$ was too similar to be distinguished (Figure 2B) [27]. However, Au and Ag can be distinguished with a high-resolution TEM technique, owing to the different atomic weights of Au and Ag (196.97 and 106.87, respectively) [28]. The dark area corresponds to Au, whereas the bright area represents Ag. As shown in Figure 2A, the Au shell has very rough surface filled with 3-5 nm sized pores.

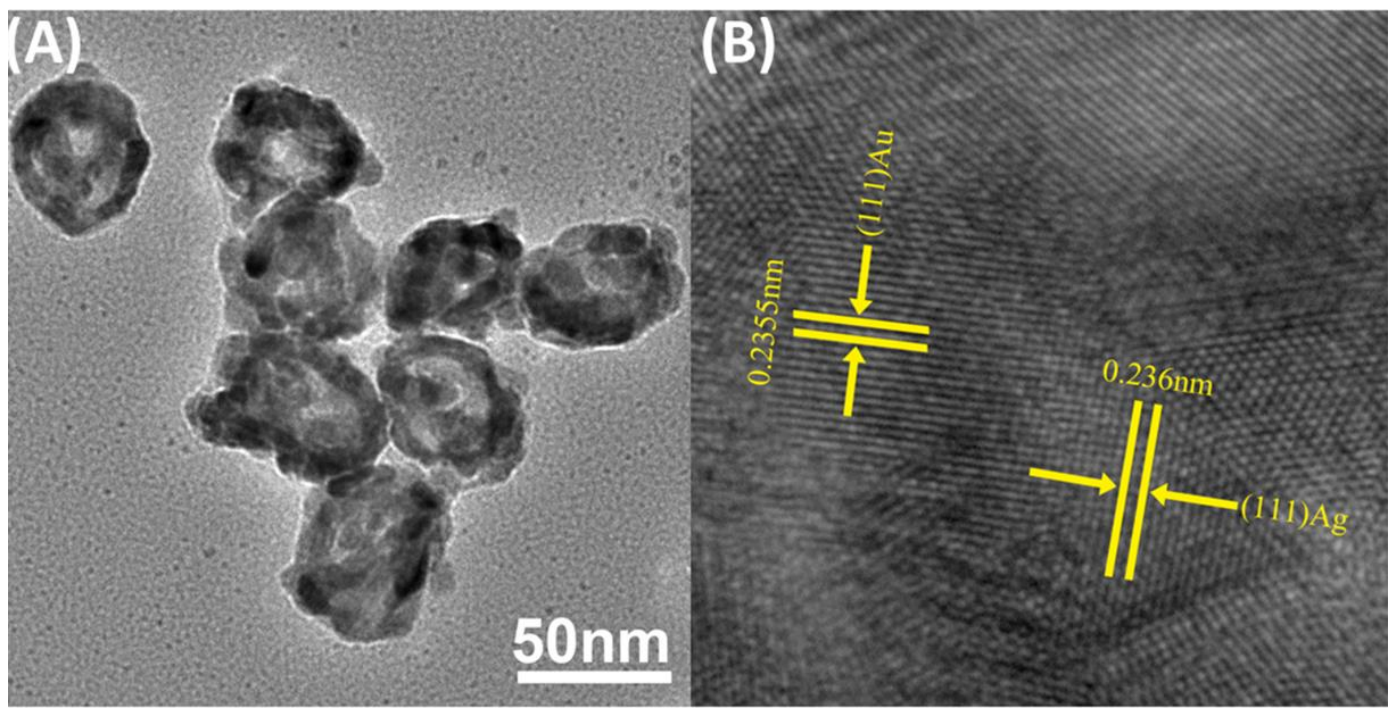

Figure 2. (A) Transmission electron microscopy (TEM) image of Ag@Au core-shell porous nanocages; (B) the lattice constant of Ag@Au core-shell porous nanocages corresponding to $1.0 \mathrm{~mL}$ of chloroauric acid aqueous solution added.

\subsection{Characterization of SERS Tags}

As shown in Scheme 1, the Ag@Au core-shell porous nanocages were first modified with 4-MBA through covalent bonding between Au and S. Then, the 4-MBA-decorated Ag@Au core-shell porous nanocages were modified by $\mathrm{Ab}_{2}$ through the reaction of $\mathrm{Au}$ and protein. The ultraviolet-visible 
(UV-vis) spectra show changes before and after the biological functionalization of Ag@Au core-shell porous nanocages (Figure 3A). The LSPR band of 50 nm Ag@Au core-shell porous nanocages was located at $450 \mathrm{~nm}$ (Figure 3A, curve a). After functionalization with 4-MBA and $\mathrm{Ab}_{2}$, the LSPR band of the nanocage SERS tags shifted to $455 \mathrm{~nm}$ while the LSPR band located at $270 \mathrm{~nm}$ appeared (Figure 3A, curve $b$ ). The above results confirmed that 4-MBA and antibodies had successfully attached to the surface of the Ag@Au core-shell porous nanocages. For comparison, Au NPs SERS tags were also synthesized (Figure 3B).
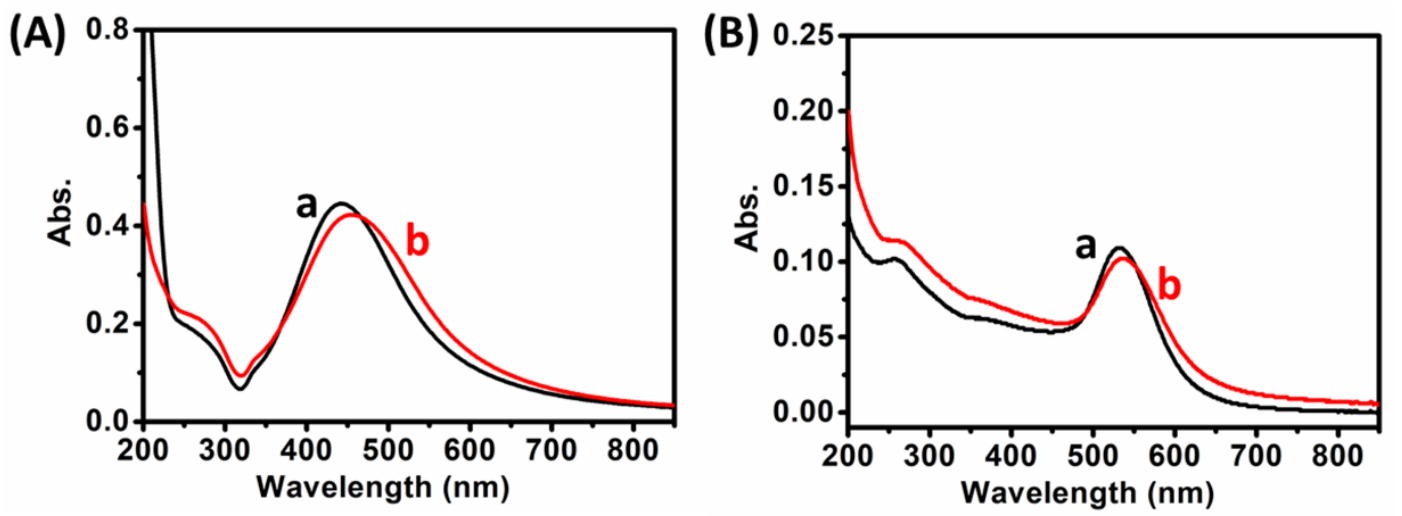

Figure 3. (A) UV-vis spectra of the hydrosols of Ag core-Au shell nanocages (a), Ag core-Au shell nanocage SERS tags (b); (B) UV-vis absorption spectra of Au (a), Au NP SERS tags (b).

Figure 4A shows that the 4-MBA-labeled Ag@Au core-shell porous nanocage SERS tags produced main SERS peaks at 716,839,1076, 1181, 1486 and $1586 \mathrm{~cm}^{-1}$. The Raman shifts at $1586 \mathrm{~cm}^{-1} \mathrm{are}^{-}$ caused by the $C=C$ stretching vibrations of 4-MBA. The Raman shifts at $1076 \mathrm{~cm}^{-1}$ are caused by the $\mathrm{C}-\mathrm{H}$ bending vibrations of 4-MBA. Results in Figure $4 \mathrm{~A}$ also illustrate that the SERS signal using Ag@Au nanocage SERS tags is more than 10 times higher than that of the Au SERS tags. This suggests that Ag@Au core-shell porous nanocages can easily outperform Au nanoparticles to make an SERS immunosensor with enormously improved sensitivity. For 15 randomly selected test points on the AFP-captured immuno-sensing chips, the intensity of the Raman peak at 4-MBA at $1076 \mathrm{~cm}^{-1} \mathrm{was}^{-}$ measured as shown in Figure $4 \mathrm{~B}$ and the relative standard deviation (RSD) of the peak intensity at each point was $6.11 \%$, indicating that the substrate had good uniformity.
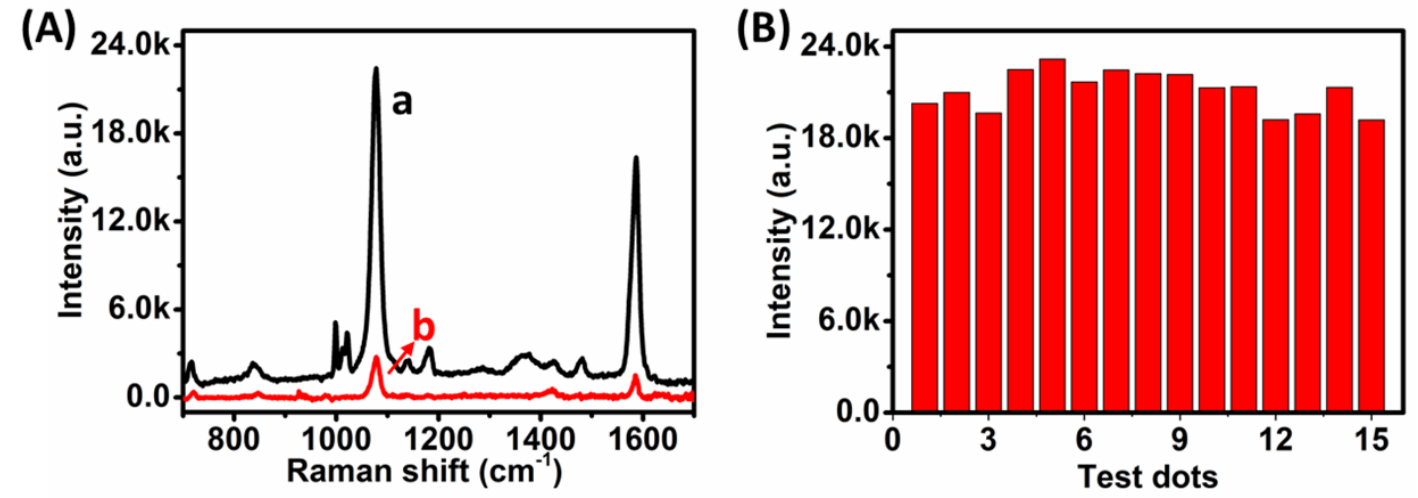

Figure 4. (A) SERS spectra of the AFP immuno-sensing chips incubated with AFP of $100 \mathrm{ng} \mathrm{mL}-1$ followed by incubation with the Ag core-Au shell nanocage SERS tags (a) and Au NP SERS tags (b), washing and drying. (B) Intensities of the SERS peak at $1076 \mathrm{~cm}^{-1}$ for 15 spots on the AFP-captured immuno-sensing chips. The excitation wavelength used was $785 \mathrm{~nm}$, the accumulation time was $20 \mathrm{~s}$ and the laser power was set to $10 \mathrm{~mW}$. 


\subsection{Optimization of the Amount of Chloroauric Acid}

In order to maximize the sensitivity of the SERS signal, the size of the Ag core and the pore and surface roughness of the Au shell were investigated in this study. As shown in Figure 5, the SERS intensity of adsorbed 4-MBA first enhanced and then weakened. When the amount of aqueous chloroauric acid solution $(0.253 \mathrm{mM})$ added to $6 \mathrm{~mL} \mathrm{Ag} \mathrm{NPs} \mathrm{was} 1.0 \mathrm{~mL}$, the SERS intensity was highest. When chloroauric acid was added into the solution of Ag NPs without any other reducing agent, corrosion appeared to commence at some specific sites of the Ag core via a galvanic replacement reaction between $\mathrm{Ag}^{0}$ and $\mathrm{AuCl}_{4}{ }^{-}$. As a result, the $\mathrm{Ag}$ core gradually became smaller, and the Au shell with a rough surface grew. This result may be attributed to the presence of pinholes in the Au layer that act as hot spots for electromagnetic field enhancement. However, the gradual depletion of the Ag core and the disappearance of pinholes resulted in a great decrease of the Raman signal. Under optimal conditions, the SERS intensity of Ag@Au core-shell porous nanocages is more than 10 times higher than that of Ag NPs or Au NPs. The higher SERS activity of the Ag@Au porous nanocages likely comes from the synergistic interactions of two factors: (1) newly generated hot spots in the rough $\mathrm{Au}$ shell for stronger plasmonic coupling and (2) the retained SERS activity of the Ag core.

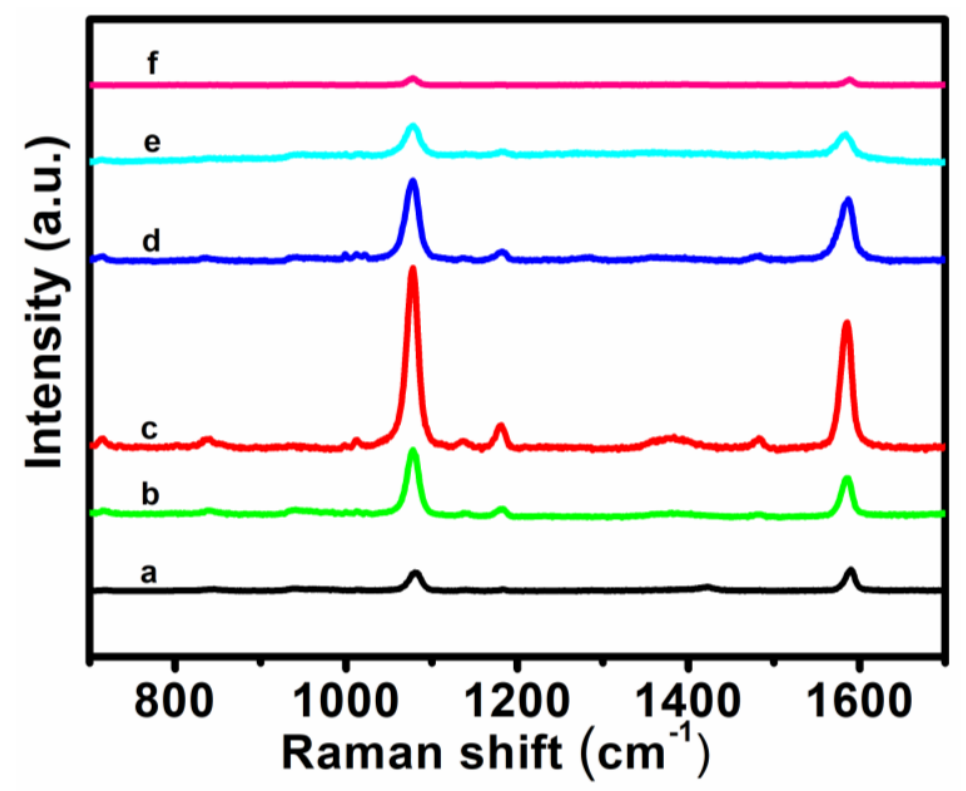

Figure 5. SERS spectra of the 4-MBA-labeled Ag core-Au shell nanocages self-assembled monolayer, corresponding to different amounts of aqueous chloroauric acid solution $(0.253 \mathrm{mM})$ added $(\mathrm{mL})$ : (a) 0.0 ; (b) 0.5 ; (c) 1.0; (d) 1.5; (e) 2.0 and (f) Au nanoparticles (NPs) for comparison. The excitation wavelength used was $785 \mathrm{~nm}$, the accumulation time was $10 \mathrm{~s}$ and the laser power was set to $10 \mathrm{~mW}$.

\subsection{Analytical Performance}

In the presence of AFP, the SERS peaks of 4-MBA from the captured SERS tags solution could be observed and the intensity increased with the AFP concentration, as demonstrated by the strongest Raman peak at $1076 \mathrm{~cm}^{-1}$ (Figure 6A). The calibration plot showed a good linear relationship between the SERS intensity and the logarithm of the analyte concentration within the range from $0.2 \mathrm{ng} \mathrm{mL}^{-1}$ to $500 \mathrm{ng} \mathrm{mL}^{-1}$ (Figure 6B). The calculated correlation coefficient was 0.998 , as shown in Figure 6B. Even at a concentration of AFP as low as $0.2 \mathrm{ng} \mathrm{mL}^{-1}$, the SERS peaks could still be directly detected without the need for any enhancement, which was much lower than that of the Au or Ag nanomaterial-based SERS immunoassay. The limit of detection (LOD) at a signal-to-noise ratio of $3 \sigma$ (where $\sigma$ is the standard deviation of signal in a blank solution) was determined to be $0.12 \mathrm{ng} \mathrm{mL}^{-1}$. 

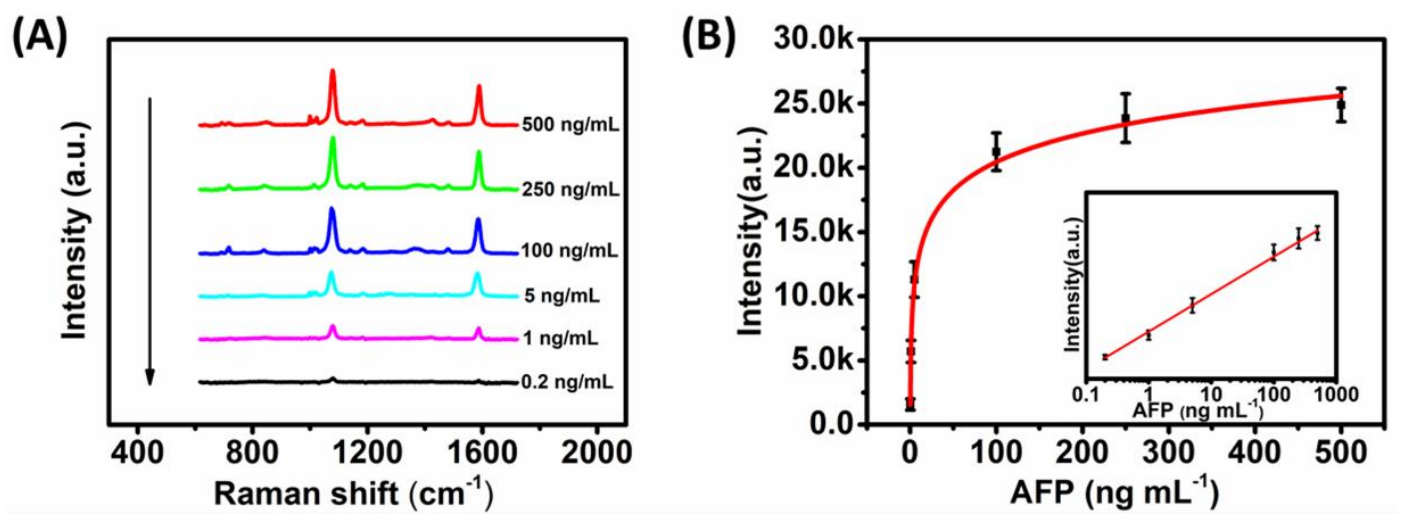

Figure 6. (A) SERS spectra of the AFP immuno-sensing chips incubated with AFP of different concentrations followed by incubation with the Ag core-Au shell nanocage SERS tags, washing and drying. (B) Intensity of the SERS peak at $1076 \mathrm{~cm}^{-1}$ as a function of the concentration of AFP. Inset shows an almost linear relationship in a logarithmic plot. The excitation wavelength used was $785 \mathrm{~nm}$, the accumulation time was $20 \mathrm{~s}$ and the laser power was set to $10 \mathrm{~mW}$.

\subsection{Reproducibility and Precision of the Immunosensor}

In order to estimate the reproducibility of the immunoassay, both the intra-assay and inter-assay precision of the immunosensor were examined with $100 \mathrm{ng} \mathrm{mL}^{-1}$ AFP five times. The RSDs were $3.2 \%$ and $3.8 \%$ (Table 1), respectively, showing good precision and acceptable fabrication reproducibility. In addition, when the immunosensor was stored in dry conditions at $4{ }^{\circ} \mathrm{C}$, over $90 \%$ of the initial response remained after a storage period of 3 weeks. These results indicated that the immunosensor had acceptable reliability and stability, and was suitable for the clinical diagnosis of protein markers.

Table 1. Intra-assay and inter-assay precisions of the immunosensor.

\begin{tabular}{|c|c|c|}
\hline \multirow[t]{2}{*}{$\begin{array}{l}100 \mathrm{ng} \mathrm{mL}^{-1} \\
\text { Sample No. }\end{array}$} & \multicolumn{2}{|c|}{$\mathrm{C}_{\mathrm{AFP}}\left(\mathrm{ng} \mathrm{mL}^{-1}\right)$} \\
\hline & Intra-Assay & Inter-Assay \\
\hline 1 & 102.6 & 102.6 \\
\hline 2 & 103.9 & 101.5 \\
\hline 3 & 97.5 & 95.9 \\
\hline 4 & 96.5 & 96.2 \\
\hline 5 & 100.6 & 104.2 \\
\hline Relative Standard Deviation (RSD) (\%) & 3.2 & 3.8 \\
\hline
\end{tabular}

\section{Conclusions}

A highly sensitive SERS immunoassay of biomarkers was developed in this research, which involves the generation of 4-MBA-labeled Ag@Au core-shell porous nanocages SERS tags and subsequent modification with AFP detection antibodies. The thus-obtained AFP immuno-sensing chips integrate the selectivity of immune recognition and overcome the drawbacks of low sensitivity and stability of Au or Ag nanoparticles. Furthermore, the Ag@Au core-shell nanocage SERS tags could be optimized to provide plenty of hot spots for achieving enormously enhanced SERS activity. Overall, this study illustrates that the Ag@Au core-shell porous nanocages can be used for highly sensitive SERS assays.

\section{Patents}

Lin, D.; Huang, Y.; Li, M.;Wang, S; Jin, H. Preparation of Ag@Au core-shell porous nanocages, surface-enhanced Raman spectroscopy detection probes and its applications. CHN. Patent CN 108580919 A, 28 September 2018. 
Author Contributions: Conceptualization, D.L. and S.W.; Data curation, Y.H.; Formal analysis, Y.H. and M.L.; Funding acquisition, D.L., S.W. and J.W.; Investigation, Y.H.; Methodology, Y.H.; Project administration, D.L. and S.W.; Supervision, S.W.; Validation, M.L.; Visualization, Y.H.; Writing—original draft, Y.H.; Writing一review and editing, D.L., D.Y., S.W. and J.W.

Funding: This research was funded by the National Natural Science Foundation of China (No.21475097 and 21471116), the Natural Science Foundation of Zhejiang Province (LY17B050004, LY18H200025 and LY17E020003) and the Opening Project of Zhejiang Provincial Top Key Discipline of Clinical Medicine (KFJ026).

Conflicts of Interest: The authors declare no conflict of interest.

\section{References}

1. Srinivas, P.R.; Kramer, B.S.; Srivastava, S. Trends in biomarker research for cancer detection. Lancet Oncol. 2001, 2, 698-704. [CrossRef]

2. Kitano, H. Systems Biology: A Brief Overview. Science 2002, 295, 1662-1664. [CrossRef] [PubMed]

3. Yuan, Y.; Yuan, R.; Chai, Y.; Zhuo, Y.; Shi, Y.; He, X.; Miao, X. A Reagentless Amperometric Immunosensor for Alpha-Fetoprotein Based on Gold Nanoparticles/ $\mathrm{TiO}_{2}$ Colloids/Prussian Blue Modified Platinum Electrode. Electroanalysis 2007, 19, 1402-1410. [CrossRef]

4. Zhao, B.; Yan, J.; Wang, D.; Ge, Z.; He, S.; He, D.; Song, S.; Fan, C. Carbon nanotubes multifunctionalized by rolling circle amplification and their application for highly sensitive detection of cancer markers. Small 2013, 9, 2595-2601. [CrossRef]

5. Chen, X.; Zhou, G.; Song, P.; Wang, J.; Gao, J.; Lu, J.; Fan, C.; Zuo, X. Ultrasensitive electrochemical detection of prostate-specific antigen by using antibodies anchored on a DNA nanostructural scaffold. Anal. Chem. 2014, 86, 7337-7342. [CrossRef]

6. Lin, D.; Mei, C.; Liu, A.; Jin, H.; Wang, S.; Wang, J. Cascade signal amplification for electrochemical immunosensing by integrating biobarcode probes, surface-initiated enzymatic polymerization and silver nanoparticle deposition. Biosens. Bioelectron. 2015, 66, 177-183. [CrossRef] [PubMed]

7. Chen, J.; Zhao, G.C. A novel signal-on photoelectrochemical immunosensor for detection of alpha-fetoprotein by in situ releasing electron donor. Biosens. Bioelectron. 2017, 98, 155-160. [CrossRef] [PubMed]

8. Zhou, L.; Ding, F.; Chen, H.; Ding, W.; Zhang, W.; Chou, S.Y. Enhancement of immunoassay's fluorescence and detection sensitivity using three-dimensional plasmonic nano-antenna-dots array. Anal. Chem. 2012, 84, 4489-4495. [CrossRef]

9. Zhang, W.; Ma, W.; Long, Y.T. Redox-mediated Indirect Fluorescence Immunoassay for the Detection of Disease Biomarkers Using Dopamine-functionalized QDs. Anal. Chem. 2016, 88, 5131-5136. [CrossRef] [PubMed]

10. Wang, H.; Ma, Z. Simultaneous detection of multiple tumor markers by label-free electrochemical immunoassay using chip-like glass carbon electrodes. Sens. Actuators B Chem. 2018, 256, 402-407. [CrossRef]

11. Dajie, L.; Jie, W.; Feng, Y.; Shengyuan, D.; Huangxian, J. Ultrasensitive immunoassay of protein biomarker based on electrochemiluminescent quenching of quantum dots by hemin bio-bar-coded nanoparticle tags. Anal. Chem. 2011, 83, 5214-5221.

12. Lei, M.A.; Sun, Y.; Kang, X.; Wan, Y. Development of nanobody-based flow injection chemiluminescence immunoassay for sensitive detection of human prealbumin. Biosens. Bioelectron. 2014, 61, 165-171. [CrossRef]

13. Pelaz, B.; Alexiou, C.; Alvarez-Puebla, R.A.; Alves, F.; Andrews, A.M.; Ashraf, S.; Balogh, L.P.; Ballerini, L.; Bestetti, A.; Brendel, C. Diverse Applications of Nanomedicine. ACS Nano 2017, 11, 2313-2381. [CrossRef] [PubMed]

14. Liu, Z.; Yang, Z.; Peng, B.; Cao, C.; Zhang, C.; You, H.; Xiong, Q.; Li, Z.; Fang, J. Highly sensitive, uniform, and reproducible surface-enhanced Raman spectroscopy from hollow Au-Ag alloy nanourchins. Adv. Mater. 2014, 26, 2431-2439. [CrossRef]

15. Lane, L.A.; Qian, X.; Nie, S. SERS Nanoparticles in Medicine: From Label-Free Detection to Spectroscopic Tagging. Chem. Rev. 2015, 115, 10489-10529. [CrossRef] [PubMed]

16. Yang, L.; Gao, M.X.; Zhan, L.; Gong, M.; Zhen, S.J.; Huang, C.Z. An enzyme-induced Au@Ag core-shell nanoStructure used for an ultrasensitive surface-enhanced Raman scattering immunoassay of cancer biomarkers. Nanoscale 2017, 9, 2640-2645. [CrossRef] 
17. Shin, K.; Cho, J.-H.; Yoon, M.-Y.; Chung, H. Use of Multiple Peptide-Based SERS Probes Binding to Different Epitopes on a Protein Biomarker to Improve Detection Sensitivity. Anal. Chem. 2016, 88, 3465-3470. [CrossRef]

18. Wang, Y.; Yan, B.; Chen, L. SERS tags: Novel optical nanoprobes for bioanalysis. Chem. Rev. 2013, 113, 1391-1428. [CrossRef] [PubMed]

19. Rodriguez-Lorenzo, L.; Krpetic, Z.; Barbosa, S.; Alvarez-Puebla, R.A.; Liz-Marzan, L.M.; Prior, I.A.; Brust, M. Intracellular mapping with SERS-encoded gold nanostars. Integr. Biol. 2011, 3, 922-926. [CrossRef]

20. Mohd Azmi, U.Z.; Yusof, N.A.; Kusnin, N.; Abdullah, J.; Suraiya, S.; Ong, P.S.; Ahmad Raston, N.H.; Abd Rahman, S.F.; Mohamad Fathil, M.F. Sandwich Electrochemical Immunosensor for Early Detection of Tuberculosis Based on Graphene/Polyaniline-Modified Screen-Printed Gold Electrode. Sensors 2018, 18, 3926. [CrossRef]

21. Cao, X.; Shan, Y.; Tan, L.; Yu, X.; Bao, M.; Li, W.; Shi, H. Hollow Au nanoflower substrates for identification and discrimination of the differentiation of bone marrow mesenchymal stem cells by surface-enhanced Raman spectroscopy. J. Mater. Chem. B 2017, 5, 5983-5995. [CrossRef]

22. Wang, W.; Wang, W.; Liu, L.; Xu, L.; Kuang, H.; Zhu, J.; Xu, C. Nanoshell-Enhanced Raman Spectroscopy on a Microplate for Staphylococcal Enterotoxin B Sensing. ACS Appl. Mater. Interfaces 2016, 8, 15591-15597. [CrossRef] [PubMed]

23. Bi, X.; Li, X.; Chen, D.; Du, X. Sensitive Glycoprotein Sandwich Assays by the Synergistic Effect of In Situ Generation of Raman Probes and Plasmonic Coupling of Ag Core-Au Satellite Nanostructures. ACS Appl. Mater. Interfaces 2016, 8, 10683-10689. [CrossRef]

24. Philip, D.; Gopchandran, K.G.; Unni, C.; Nissamudeen, K.M. Synthesis, characterization and SERS activity of Au-Ag nanorods. Spectrochim. Acta A Mol. Biomol. Spectrosc. 2008, 70, 780-784. [CrossRef] [PubMed]

25. Hao, E.; Li, S.; Bailey, R.C.; Zou, S.; Schatz, G.C.; Hupp, J.T. Optical Properties of Metal Nanoshells. J. Phys. Chem. B 2004, 108, 1224-1229. [CrossRef]

26. Li, H.; Xia, H.; Ding, W.; Li, Y.; Shi, Q.; Wang, D.; Tao, X. Synthesis of monodisperse, quasi-spherical silver nanoparticles with sizes defined by the nature of silver precursors. Langmuir 2014, 30, 2498-2504. [CrossRef]

27. Srnová-Šloufová, I.; Lednický, F.; Gemperle, A.; Gemperlová, J. Core-Shell (Ag)Au Bimetallic Nanoparticles: Analysis of Transmission Electron Microscopy Images. Langmuir 2000, 16, 9928-9935. [CrossRef]

28. Fu, H.; Yang, X.; Jiang, X.; Yu, A. Bimetallic Ag-Au nanowires: Synthesis, growth mechanism, and catalytic properties. Langmuir 2013, 29, 7134-7142. [CrossRef] [PubMed] 